\title{
REVIEW
}

Open Access

\section{Systematic review and meta-analysis of maintenance of physical activity behaviour change in cancer survivors}

Chloe Grimmett $^{1 *}$ (D), Teresa Corbett ${ }^{1}$, Jennifer Brunet ${ }^{3}$, Jonathan Shepherd ${ }^{2}$, Bernardine M. Pinto ${ }^{4}$, Carl R. May ${ }^{5}$ and Claire Foster ${ }^{1}$

\begin{abstract}
Background: Physical activity can improve health and wellbeing after cancer and may reduce cancer recurrence and mortality. To achieve such long-term benefits cancer survivors must be habitually active. This review evaluates the effectiveness of interventions in supporting maintenance of physical activity behaviour change among adults diagnosed with cancer and explores which intervention components and contextual features are associated with effectiveness.
\end{abstract}

Methods: Relevant randomised controlled trials (RCTs) were identified by a search of Ovid Medline, Ovid Embase and PsychINFO. Trials including adults diagnosed with cancer, assessed an intervention targeting physical activity and reported physical activity behaviour at baseline and $\geq 3$ months post-intervention were included. The behaviour change technique (BCT) taxonomy was used to identify intervention components and the Template for Intervention Description and Replication to capture contextual features. Random effect meta-analysis explored between and within group differences in physical activity behaviour. Standardised mean differences (SMD) describe effect size.

Results: Twenty seven RCTs were included, 19 were pooled in meta-analyses. Interventions were effective at changing long-term behaviour; SMD in moderate to vigorous physical activity (MVPA) between groups 0.25; 95\% $\mathrm{Cl}=0.16-0.35$. Within-group pre-post intervention analysis yielded a mean increase of $27.48(95 \% \mathrm{Cl}=11.48-43$. 49) mins/wk. of MVPA in control groups and $65.30(95 \% \mathrm{Cl}=45.59-85.01)$ mins/wk. of MVPA in intervention groups. Ineffective interventions tended to include older populations with existing physical limitations, had fewer contacts with participants, were less likely to include a supervised element or the BCTs of 'action planning', 'graded tasks' and 'social support (unspecified)'. Included studies were biased towards inclusion of younger, female, well-educated and white populations who were already engaging in some physical activity.

Conclusions: Existing interventions are effective in achieving modest increases in physical activity at least 3 months post-intervention completion. Small improvements were also evident in control groups suggesting lowintensity interventions may be sufficient in promoting small changes in behaviour that last beyond intervention completion. However, study samples are not representative of typical cancer populations. Interventions should consider a stepped-care approach, providing more intensive support for older people with physical limitations and others less likely to engage in these interventions.

Keywords: Systematic review, Meta-analysis, Physical activity, Exercise, Cancer, Behaviour change, Behaviour change techniques, Maintenance, Intervention

\footnotetext{
* Correspondence: c.grimmett@soton.ac.uk

${ }^{1}$ School of Health Sciences, University of Southampton, Southampton, UK

Full list of author information is available at the end of the article
}

(c) The Author(s). 2019 Open Access This article is distributed under the terms of the Creative Commons Attribution 4.0 International License (http://creativecommons.org/licenses/by/4.0/), which permits unrestricted use, distribution, and reproduction in any medium, provided you give appropriate credit to the original author(s) and the source, provide a link to the Creative Commons license, and indicate if changes were made. The Creative Commons Public Domain Dedication waiver (http://creativecommons.org/publicdomain/zero/1.0/) applies to the data made available in this article, unless otherwise stated. 


\section{Background}

The incidence of cancer is increasing worldwide with an estimated 14.1 million diagnoses in 2012 and projections of 23.6 million new cases each year by 2030 [1]. With advances in early diagnosis and treatments survival rates are increasing. Thirty-two million people globally were alive at least 5 years following a diagnosis in 2012 [2]. Such advances are applauded, but the disease and its treatment can have long-term impact on a person's physical and psychological health, including declines in physical function, cancer-related fatigue and poor quality of life [3].

There is good evidence that regular physical activity can improve many negative consequences reported by adults diagnosed with cancer $[4,5]$. It is also well established that regular physical activity reduces the risk of developing comorbid disease, and delays decline in physical and mental functioning associated with aging [6]. Furthermore, accumulating observational evidence suggests regular physical activity may reduce cancer recurrence and mortality and prolong disease-free survival [7-10].

Consequently, adult cancer survivors have much to gain from being regularly active and it is recommended that they meet physical activity guidelines, that is to avoid inactivity and participate in at least $150 \mathrm{~min}$ of moderate intensity physical activity (or equivalent of vigorous activity) and two sessions of resistance exercise each week $[11,12]$. Perhaps unsurprisingly, few cancer survivors meet these recommendations [13, 14] and they are less active than those without a history of the disease $[15,16]$.

Researchers in behavioural science are endeavouring to design interventions that promote regular physical activity among this population. Empirical evidence from a growing number of randomised controlled trials (RCT) suggests successful increases in physical activity on completion of interventions. In a meta-analysis of 12 interventions based on social cognitive theory, Stacey et al. [17] report a significant intervention effect for physical activity $(\mathrm{SMD}=0.33 ; p<0.01)$. Similarly, a systematic review and meta-analysis of 14 interventions designed to promote physical activity behaviour change in breast cancer survivors concluded that many interventions were effective in producing short-term improvements in physical activity [18].

However, to achieve long-term health benefits, behaviour change must be sustained and we know much less about effectiveness of interventions for promoting long-term change. For example, in a review of the maintenance of outcomes following physical activity and/or dietary interventions in breast cancer survivors only 10 (16\%) of the 63 trials identified assessed outcomes at least 3 months after the intervention had ended. The majority of outcomes were physical or psychosocial variables, such as functional status, cancer-related fatigue and quality of life rather than measures of physical activity [19]. Jankowski et al. [20] synthesised the evidence of maintenance of outcomes, albeit not limited to physical activity behaviour, following physical activity interventions among cancer survivors in the hope of informing translation of research into practice within community settings. Only 12 RCTs reporting maintenance of intervention outcomes were identified, four included data on minutes of physical activity with two achieving significant improvements in behaviour.

Additionally, little is known about intervention components that might facilitate long-term behaviour change. Behaviour change interventions are frequently complex, consisting of numerous interacting components that are often poorly described [21]. This makes synthesising the evidence for effectiveness to inform future interventions challenging. In order to advance behavioural medicine by promoting precise and consistent reporting of complex interventions, Michie and colleagues [22] developed The Behaviour Change Technique Taxonomy (version 1). Based on expert consensus, this hierarchical classification system has been used in numerous systematic reviews to reliably identify behaviour change techniques associated with the most successful interventions (e.g. [23-25]. Coding the functions of existing interventions and the behaviour change techniques employed, and comparing these components across effective and ineffective interventions may help to identify the successful 'active ingredients' of interventions [26].

In addition to the contribution of Michie et al's [22] theory-based BCT taxonomy, guidelines have been developed to improve reporting of interventions. The Template for Intervention Description and Replication (TIDieR) checklist highlights numerous contextual factors of intervention delivery which may impact intervention efficacy that the BCT taxonomy does not capture. Examples include mode of intervention delivery, such as home-based programs verses on-site supervised activity sessions, the intensity and duration of the intervention, and fidelity assessment [27]. Synthesis of both intervention components (through coding of BCTs) and intervention context (through the TIDieR checklist) and their association with longer-term physical activity behaviour could help inform development of future programmes.

When attempting to understand the optimal methods to support physical behaviour change, examination of RCTs has limitations. By only evaluating an intervention as 'successful' if there are statistically significant differences between intervention and control/comparison groups at follow-up we may ignore important changes in the control groups. It is well established that 
'contamination' by control participants is common in trials of physical activity and dietary behaviour change [28]. Those who agree to participate are often highly motivated to make positive change, and thus may modify their behaviour irrespective of group allocation. This can lead to type II error, that is, an intervention being perceived as ineffective even when large increases in behaviour are achieved in the intervention group. This issue is then magnified by pooling such data in meta-analysis of RCTs. Furthermore, by relying on between group differences as a marker of success and then exploring intervention components and sample characteristics in order to explain the superiority of these interventions, we risk reaching erroneous conclusions as to what components might contribute to the most effective interventions. Trials of behaviour change often include 'attention control' designs and/or provide readily available printed materials promoting engagement in regular physical activity. By quantifying change in long-term behaviour in control groups, we get a sense of the amount of behaviour change elicited by these processes and by the act of consenting to a trial of physical activity in the context of cancer and recovery.

In this paper we describe the first reported systematic review and meta-analysis of RCTs in adults affected by any type of cancer, evaluating the efficacy of interventions on maintenance of physical activity behaviour change. Using the BCT taxonomy (v1) and TIDieR checklist, we attempt to identify both intervention components and contextual features that are associated with successful, post-intervention behaviour change to inform future intervention development. Meta-analysis of long-term change in physical activity behaviour in control groups is also presented and discussed.

\section{Methods}

Guidance from the Centre for Reviews and Dissemination [29] and the Preferred Reporting Items for Systematic Reviews and Meta-analysis (PRISMA) [30] informed the methods for conducting and reporting this review. This review was registered with PROSPERO; CRD42017068924.

\section{Literature searching}

The following databases were systematically searched from inception to August 2018: Ovid Medline, Epub ahead of print, In Process \& Other non-indexed citations, Ovid Embase and Ebsco PsycINFO. Conference proceedings were searched from 2015 to August 2018 via the Web of Science platform Science Citation Index \& Social Science Citation Index \& Conference Proceedings Citation Index. The strategy is a balanced combination of index and free text synonym terms, representing cancer survivor, exercise, physical activity, and lifestyle interventions. A clinical trial search filter was applied to the strategy to identify randomized or controlled trials or validation or evaluation studies. The search was limited to studies published in English. Reference lists of all included articles were hand searched for other relevant papers. The search results were screened on title and abstract against the inclusion criteria by two researchers (CG and TC). Full texts were obtained for publications identified to be potentially relevant and were screened independently by two reviewers (CG and TC); disagreements were discussed until a consensus was reached. Search hit returns can be referred to in the PRISMA chart (Fig. 1.). An example search strategy (Medline) is listed in Additional file 1.

\section{Study selection}

The eligibility criteria for study inclusion were; 1 ) trials including adults ( $\geq 18$ years) with a diagnosis of cancer, 2 ) trials that assessed an intervention that targeted aerobic physical activity (interventions limited to exercises for specific areas of the body, such as arm exercises for lymphoedema or pelvic floor exercises were excluded), 3) participants were randomised to an intervention and a control/comparison group, 4) reported outcomes data on physical activity behaviour (this could be expressed as an estimate of total energy expenditure (e.g. calories per kilogram per week (kcal/kg/week), METs per week (METs/wk) or minutes per week (mins/wk) of moderate to vigorous physical activity (MVPA) at the end of intervention measured directly or indirectly, and at least 3 months post-intervention completion with no intervention contact between the end of intervention and post-intervention follow-up. With no clear consensus on the definition of 'maintenance' of a behavioural outcome, the criterion of at least 3 months was selected as per previous systematic reviews of physical activity among cancer populations [31, 32]. The original protocol specified inclusion of pre-post studies however this was modified to include RCTs only to ensure optimal methodological rigour.

\section{Data extraction and risk of bias assessment}

A data extraction form capturing study details and physical activity behaviour data was developed and iteratively refined by CG to ensure comprehensive data capture. One author (CG) extracted data on author, country of study, study design, sample size, population studied (including cancer type, age, comorbidities, ethnicity and level of education), intervention type (i.e. physical activity only or lifestyle intervention that was not restricted to physical activity), study duration, attrition rate, physical activity outcome measure and physical activity data. Features of the intervention were extracted based on the TIDieR checklist for reporting of interventions. Theoretical basis was also captured. The BCT taxonomy (v1) 


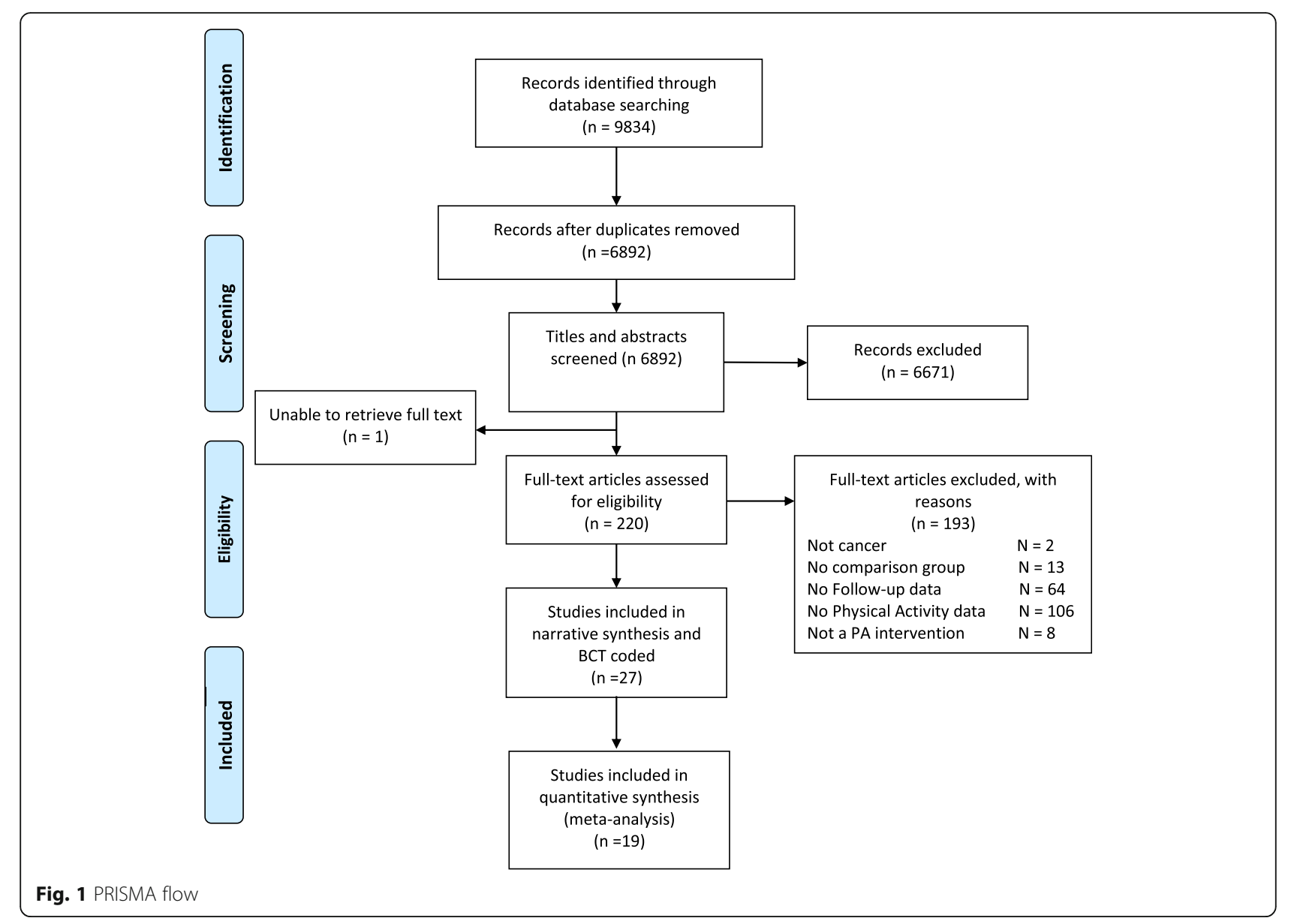

was used to code BCTs based on information presented in the included papers, as well as any published protocol papers. Two authors (CG, TC) independently coded $\mathrm{BCTs}$ for all included studies. Discrepancies were resolved through discussion.

The risk of bias of the studies included in the meta-analysis was assessed by CG and TC using the Cochrane Collaboration's risk of bias tool [33]. Six different sources of bias were considered: selection bias, performance bias, detection bias, attrition bias, reporting bias, and other bias. Risk of bias was described as 'low', 'high' or 'unclear'. Scoring conflicts were discussed and resolved by three authors (CG, TC, JS).

\section{Statistical methods}

\section{Between group differences in physical activity}

Studies reporting total minutes of MVPA per week for control and experimental groups at baseline and post-intervention follow-up were included in a meta-analysis using Cochrane Review Manager 5.3 software [34]. Guidance for pooling data for meta-analysis recommends combining data that are as similar as possible, therefore trials reporting physical activity by other means (e.g. METs/wk., kcal/kg/day, walking time and $\mathrm{kcal} /$ week) were not included. The difference in MVPA mins/wk. between the control and intervention groups at the last post-intervention follow-up assessment were used to calculate effect size, and intention-to-treat data were used when available. When insufficient data were available for the purposes of meta-analysis (e.g. no standard deviation (SD) or unadjusted baseline data presented), authors were contacted to provide the data required. If standard errors or confidence intervals were presented instead of SD, we calculated the SDs. The vast majority of studies included a self-report measure of MVPA, therefore these data were used. Data from objective measures of physical activity were included if they were available. For studies reporting moderate and vigorous activity separately, combined MVPA and SDs were calculated using the following formula, as per [35]:

$$
\bar{x}_{M V P A}=\bar{x}_{\text {moderate } P A}+\bar{x}_{\text {vigorous } P A}
$$

To combine SDs, the following formula was used 


$$
\sigma_{M V P A}=\sqrt{\left(\left(\sigma_{\text {moderate PA }}^{2}\right)+\left(\sigma_{\text {vigorous } P A}^{2}\right)\right)}
$$

A random effects model was used to calculate standardized mean difference (SMD) with $95 \%$ confidence intervals. A random effects model was chosen as interventions and outcome measures of physical activity varied widely and this type of model is recommended where heterogeneity is suspected.

Post-hoc sensitivity analysis was performed to include only those studies with $>6$ month follow-up data (a more conservative definition of maintenance of behaviour).

\section{Pre-post change in physical activity within control group and intervention group}

The change from baseline to post-intervention follow-up in minutes of MVPA per week within control and intervention groups was estimated separately in meta-analysis. As above, random effects models were used due to heterogeneity in interventions and outcomes measures.

Random effects models were also used to calculate mean difference with $95 \%$ confidence intervals for between and within group analysis to provide mean difference/change in MVPA mins/wk. to aid clinical interpretation.

Statistical approaches to explore associations between study population and intervention characteristics (including BCTs) and intervention effects e.g. meta-analysis, such as meta-regression, were considered. However, due to the relatively small number of included studies, these were not deemed feasible. Therefore, a narrative synthesis of these factors is presented using a similar categorisation methodology to Gardner et al. [36]. Trials were categorised as 'very promising,' 'quite promising' or 'not promising'. Category allocation was dependent on within or between group differences in physical activity behaviour at post-intervention follow-up. 'Very promising' trials included those with statistically significant between group differences in MVPA mins/wk. at post-intervention follow-up. 'Quite promising' were trials reporting within-group differences in the intervention group at post-intervention follow-up and 'not promising' were those that reported neither within nor between group differences at post-intervention follow-up. These categories are mutually exclusive. This categorisation enabled data interpretation and narrative synthesis of intervention and context, associated with the most and least promising interventions. In order to recognise intervention components that were associated with effectiveness, we identified BCTs that were common, defined as appearing in at least half, of the 'very promising' and 'quite promising' studies, and were uncommon, defined as appearing in less than half, of the 'not promising' studies.

\section{Results}

Figure 1 shows the flow of studies for this review. Twenty seven studies met the inclusion criteria and were included in this review.

Figure 2 presents the risk of bias assessment of studies included in the meta-analyses. Random sequence generation and allocation concealment was reasonably well reported however the majority of studies did not demonstrate blinding of outcome assessments and a number were limited by attrition bias. Most studies were also judged as 'high risk' for other sources of bias with many using self-reported physical activity measures which is prone to bias.

Table 1 presents the characteristics of the populations from each study. Studies were published between 2006 and 2018 and more than half $(15 / 27)$ were conducted in the USA and Canada. Sample sizes ranged from 41 [37] - 641 participants [38]. Most studies (18/27) included women diagnosed with breast cancer and participants were relatively young (average age 58.2 years) and well educated with $69.7 \%$ participating in some higher education. Number of comorbidities was infrequently reported and where data were available considerable variation was evident, ranging from more than $90 \%$ of participants reporting at least one comorbidity [39], to less than $30 \%$ reporting at least one comorbidity [40]. Table 2 provides data on intervention characteristics and context. Intervention modality varied from 'light touch' approaches such as providing printed materials only to more intensive designs including supervised exercise sessions and regular follow-ups, predominantly by telephone. The length of the interventions varied from a single contact [41] to 10 months of regular interactions [38]. Length of post-intervention follow-up ranged from 3 months to 5 years.

After obtaining data from authors of 7 of the included studies [42-48] 19 studies were eligible to be included in the between group meta-analysis; of these, 12 studies excluded participants who were already meeting physically active guidelines, however the median baseline levels of physical activity in the intervention groups was 86 mins/ wk. of MVPA, range 23.5 (44)-879 [48].

Two of the included studies presented physical activity data on a subsample of participants. Ottenbacher et al. [44] presented analysis for participants in the FRESH START trial who failed to meet physical activity guidelines (< 150 mins MVPA/wk) on study entry: 400 of the 543 breast and prostate cancer survivors enrolled in this home-based lifestyle intervention. Belanger et al. [40] provided data for the 96 of the 212 young adult cancer survivors who were engaging in $\leq 300 \mathrm{~min}$ of physical 


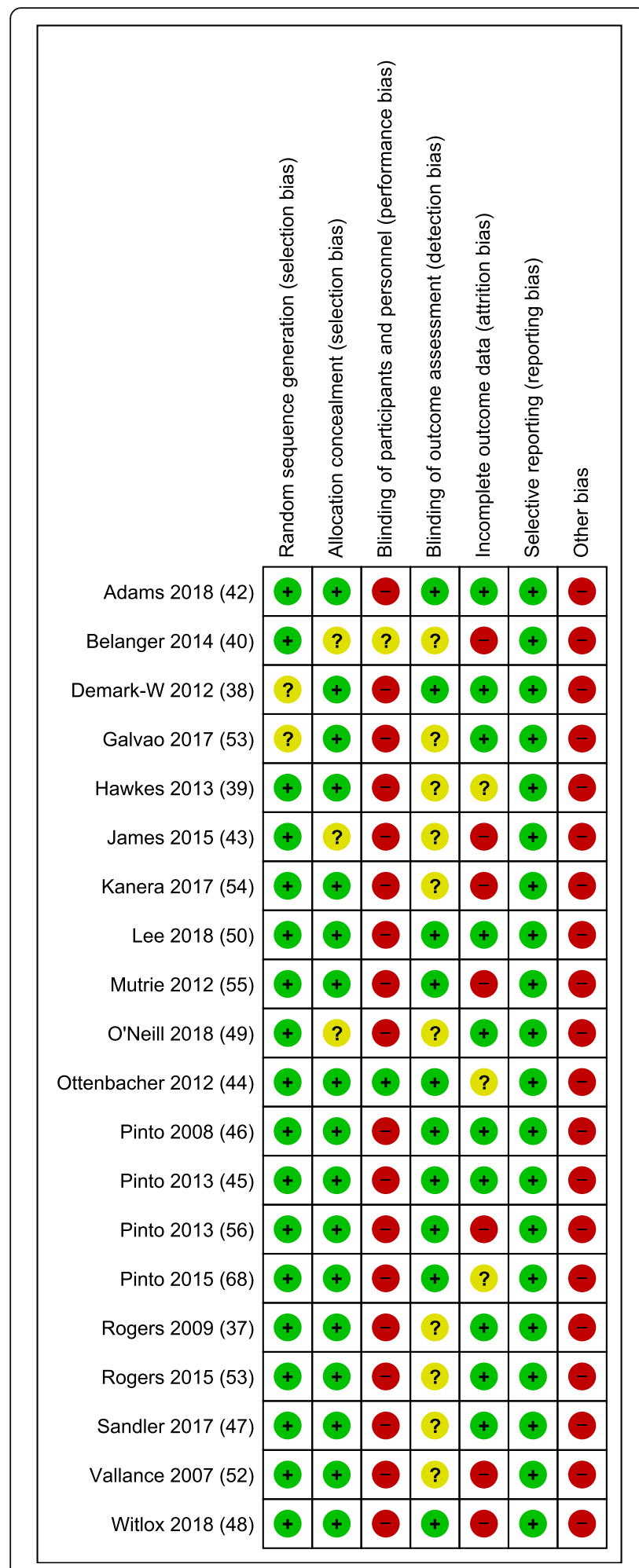

Fig. 2 Risk of Bias Assessment activity at baseline. These data were included in the meta-analysis.

\section{Behaviour change outcomes}

Nineteen of the 27 included studies provided PA data as MVPA mins/wk. data for the experimental and control groups at the post-intervention follow-up and were included in the meta-analysis. All but three studies presented data on self-reported levels of physical activity, Rogers et al. [37], O’Neil et al. [49] and Lee et al. [50] reported only objectively measured physical activity using accelerometry. Two studies included more than one intervention group $[51,52]$. In both instances the most intensive intervention was compared to the control group in meta-analyses. Figure 3 presents a forest plot detailing the SMD with 95\% confidence intervals (CIs) for the post-intervention MVPA mins/wk. data. The SMD between groups favoured the intervention group with a small estimated effect $(0.25$; $(95 \% \mathrm{CI}=0.16-0.35))$ and moderate statistical heterogeneity $\left(\mathrm{I}^{2}=36 \%\right)$. The mean difference between the intervention and controls groups was 39.88 (95\% CI $=22.78-56.97)$ MVPA mins/wk., $p<0.01$.

\section{Post-hoc sensitivity analysis}

Post-hoc sensitivity analysis was conducted with the 10 studies that presented data on MVPA mins/wk. a minimum of 6 months after intervention completion $[39,44,45,48,50,53-57]$. A very similar SMD was found; $0.21 ; 95 \% \mathrm{CI}=0.12-0.29, \mathrm{p}<0.01, \mathrm{I}^{2}=13 \%$.

\section{Within group meta-analysis}

Within group pre-post intervention analyses of physical activity behaviour in control groups reveals a small SMD: 0.21 (95\% CI =0.08-0.35), $P<0.01, \mathrm{I}^{2}=70 \%$. See Fig. 4. Mean difference $=27.48(95 \% \mathrm{CI}=11.48-43.49)$ mins/wk. of MVPA, $\mathrm{p}<0.01$.

Within group analyses of physical activity behaviour in the intervention groups revealed a moderate SMD 0.49 $(95 \%=$ CI $0.32-0.66), \mathrm{p}<0.01$, with high heterogeneity $\left(\mathrm{I}^{2}=83 \%\right)$ as shown in Fig. 5. The mean difference between baseline and post-intervention follow-up was $65.30(95 \% \mathrm{CI}=45.59-85.01) \mathrm{mins} / \mathrm{wk}$. of MVPA. Of note, one additional trial was included in this analysis [38]. Demark-Wahnefried et al. (2012) provided baseline and post-intervention (2 year) follow-up for the intervention group. The comparison group were offered the intervention at 1 year and were therefore excluded from all other meta-analyses.

As the correlation between objective and self-reported physical activity levels is often poor, all meta-analyses were re-run in a post-hoc sensitivity analysis removing the 3 studies that used objective measures of physical activity; this had negligible impact on the results: between group SMD: 0.27 (95\% 


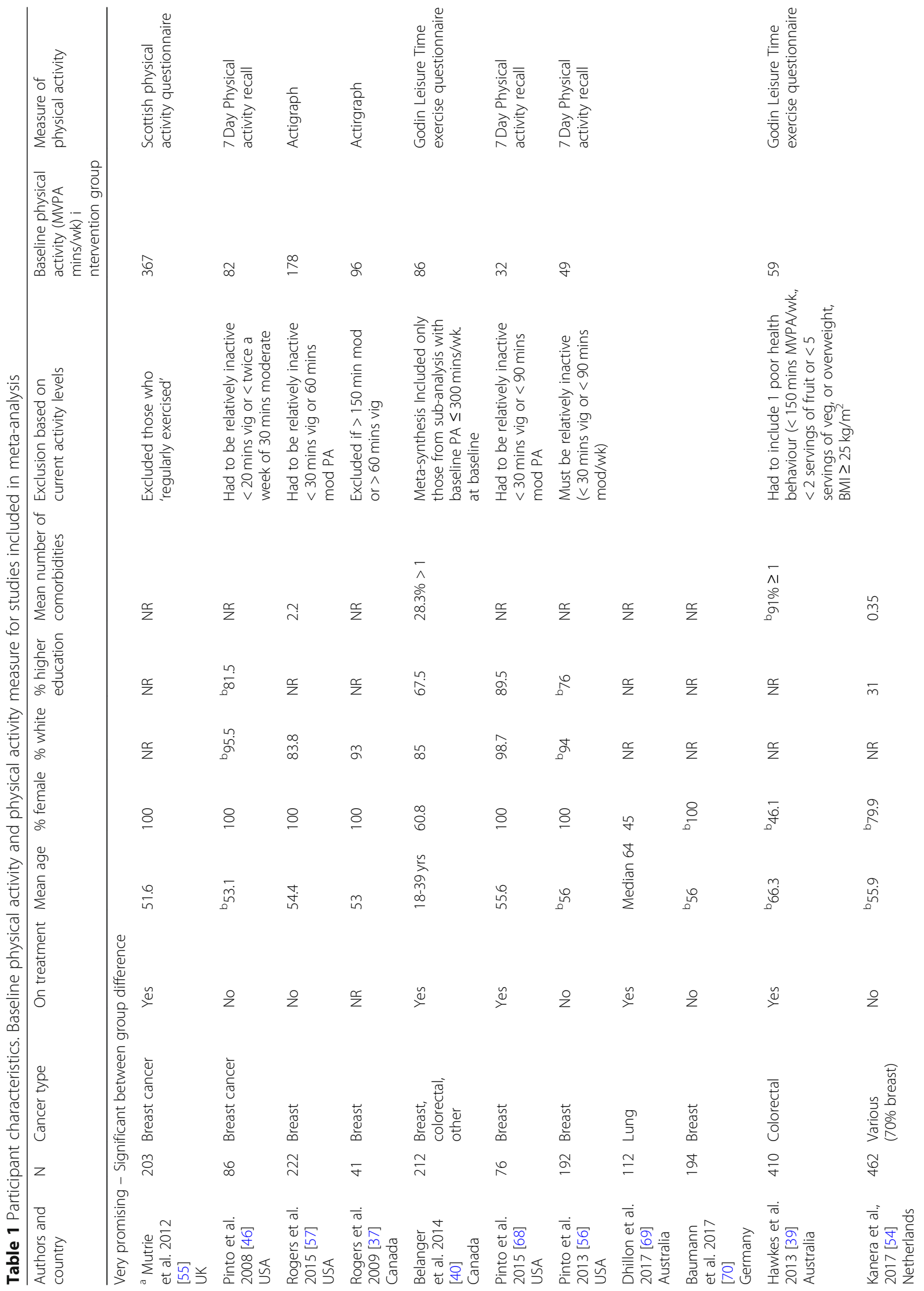




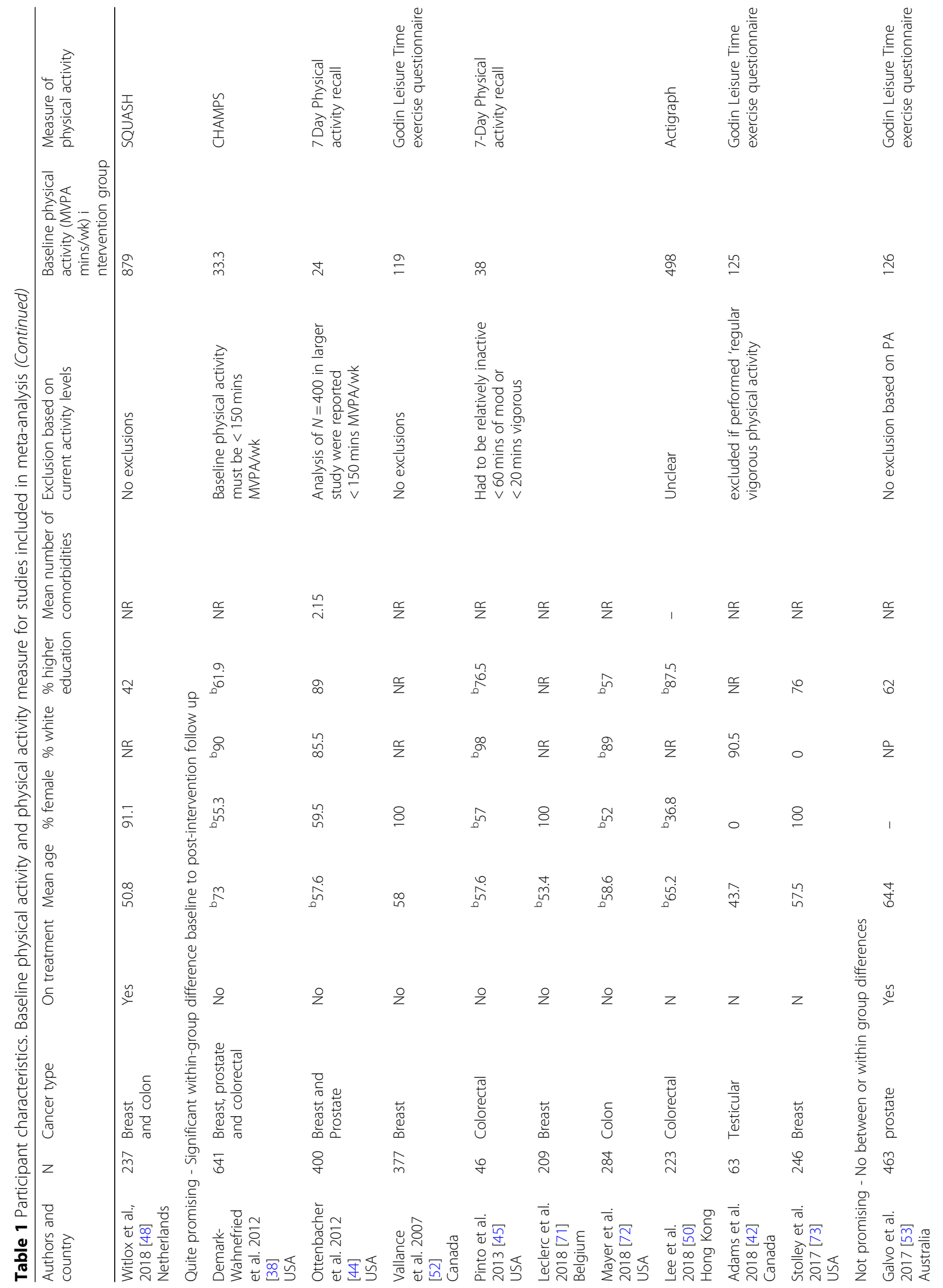




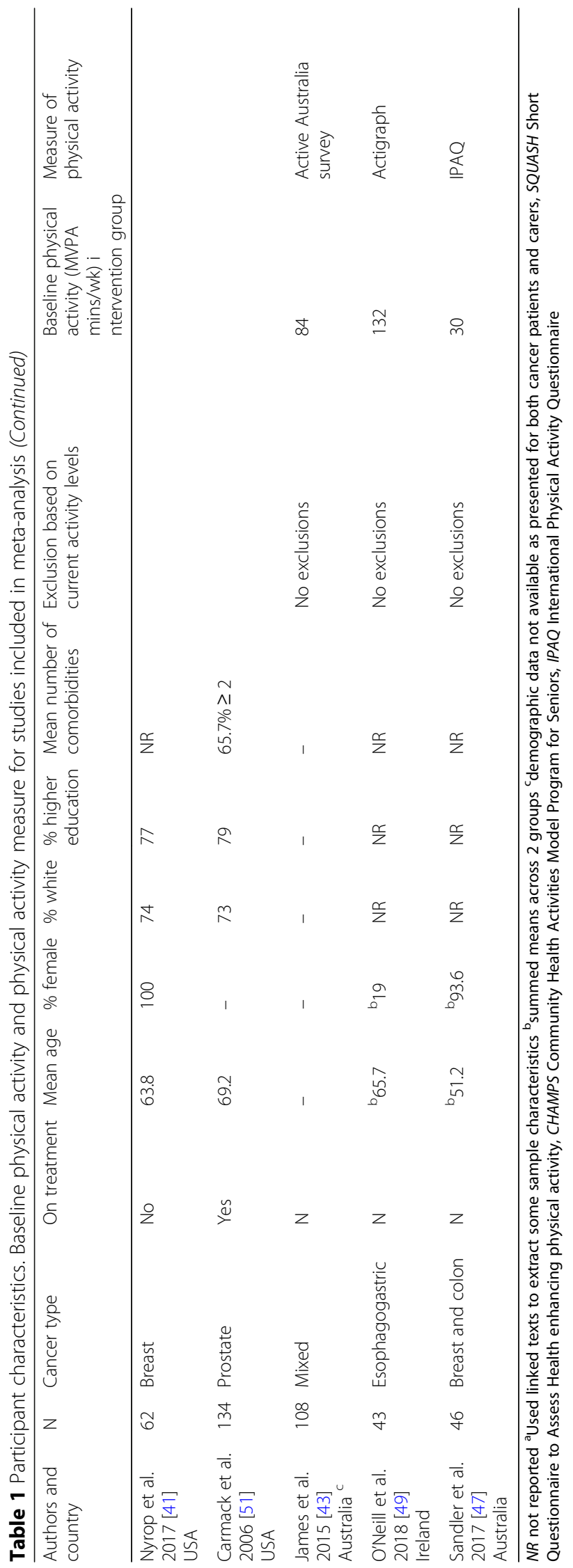




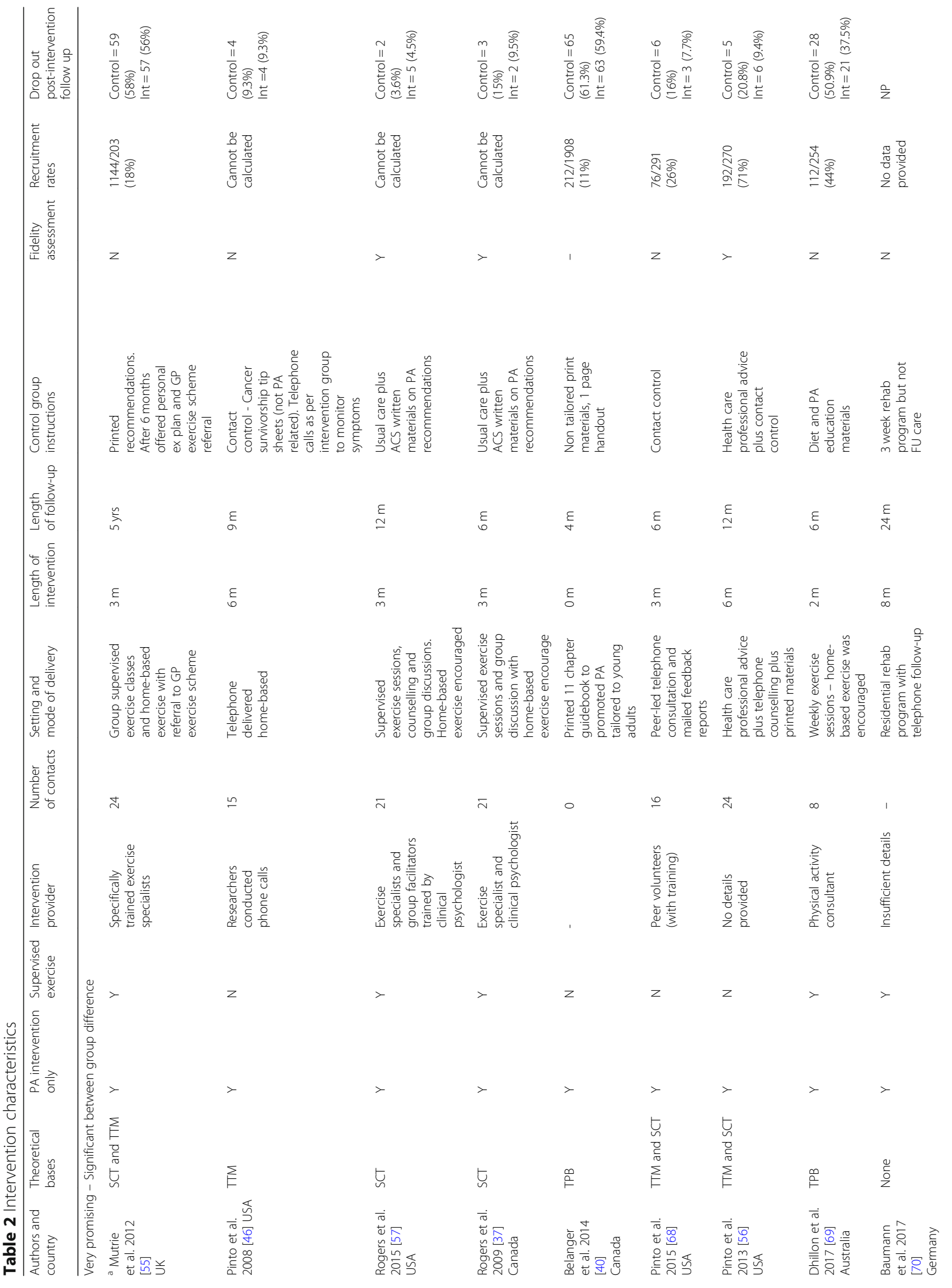


Grimmett et al. International Journal of Behavioral Nutrition and Physical Activity

(2019) $16: 37$

Page 11 of 20

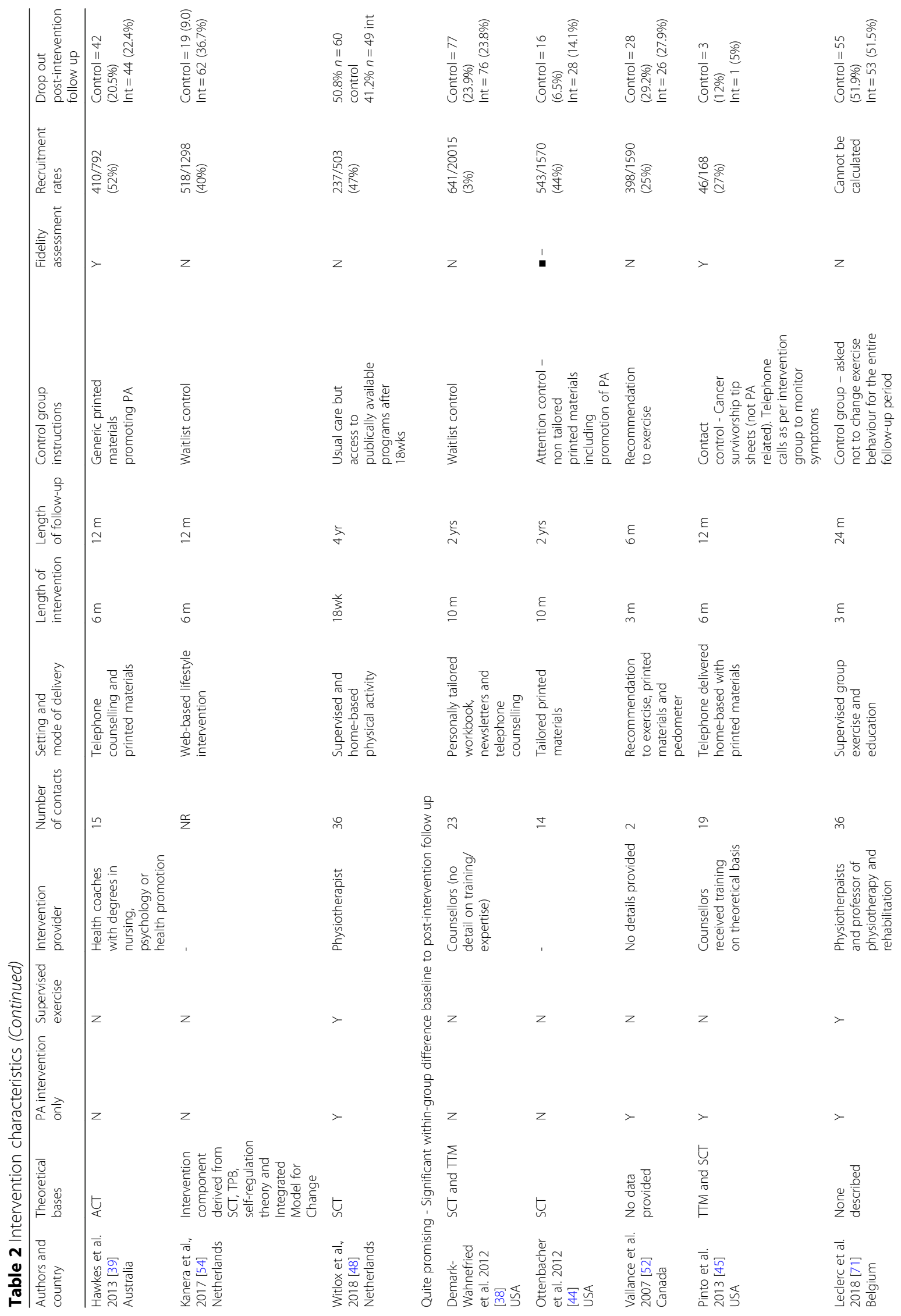




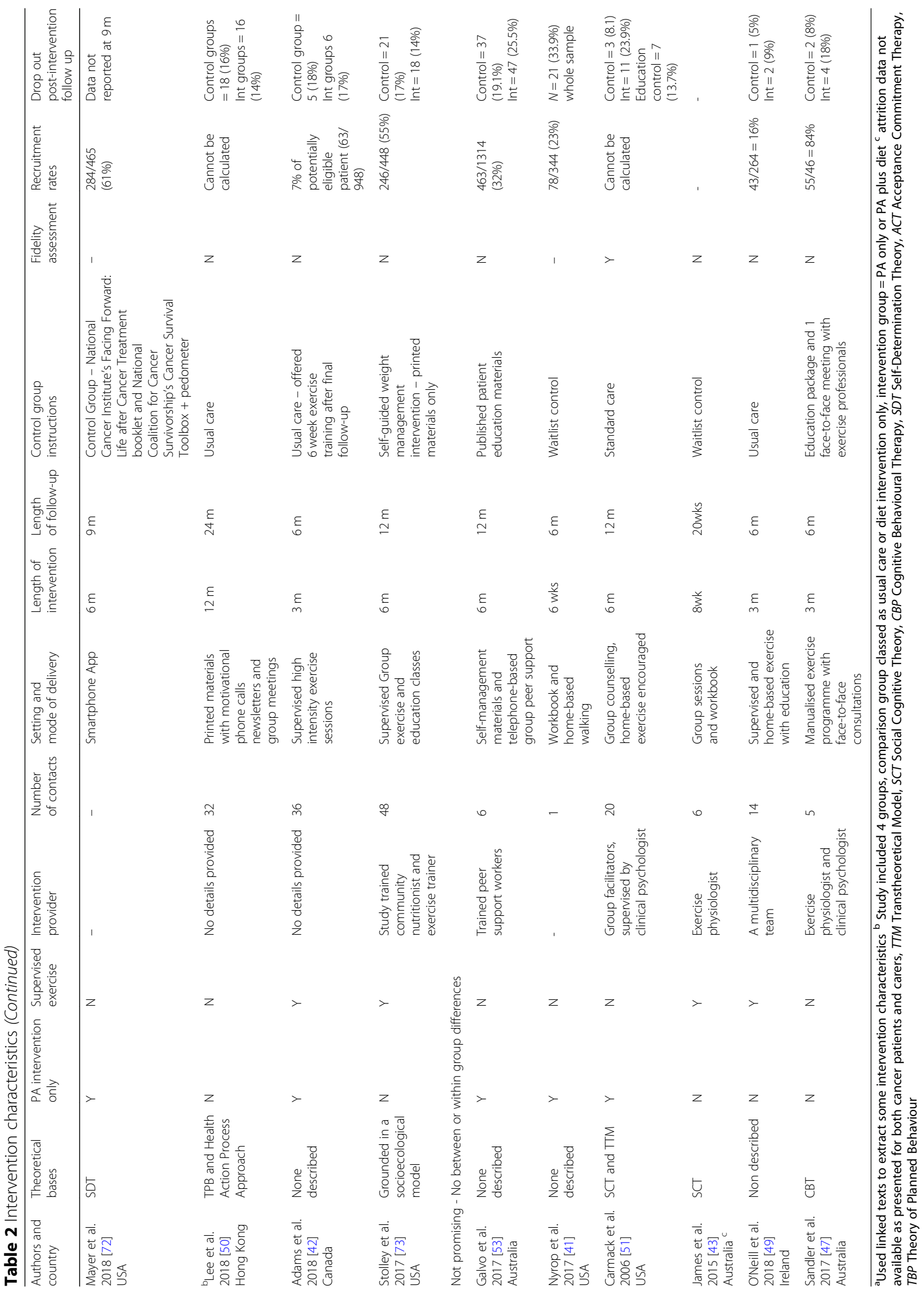




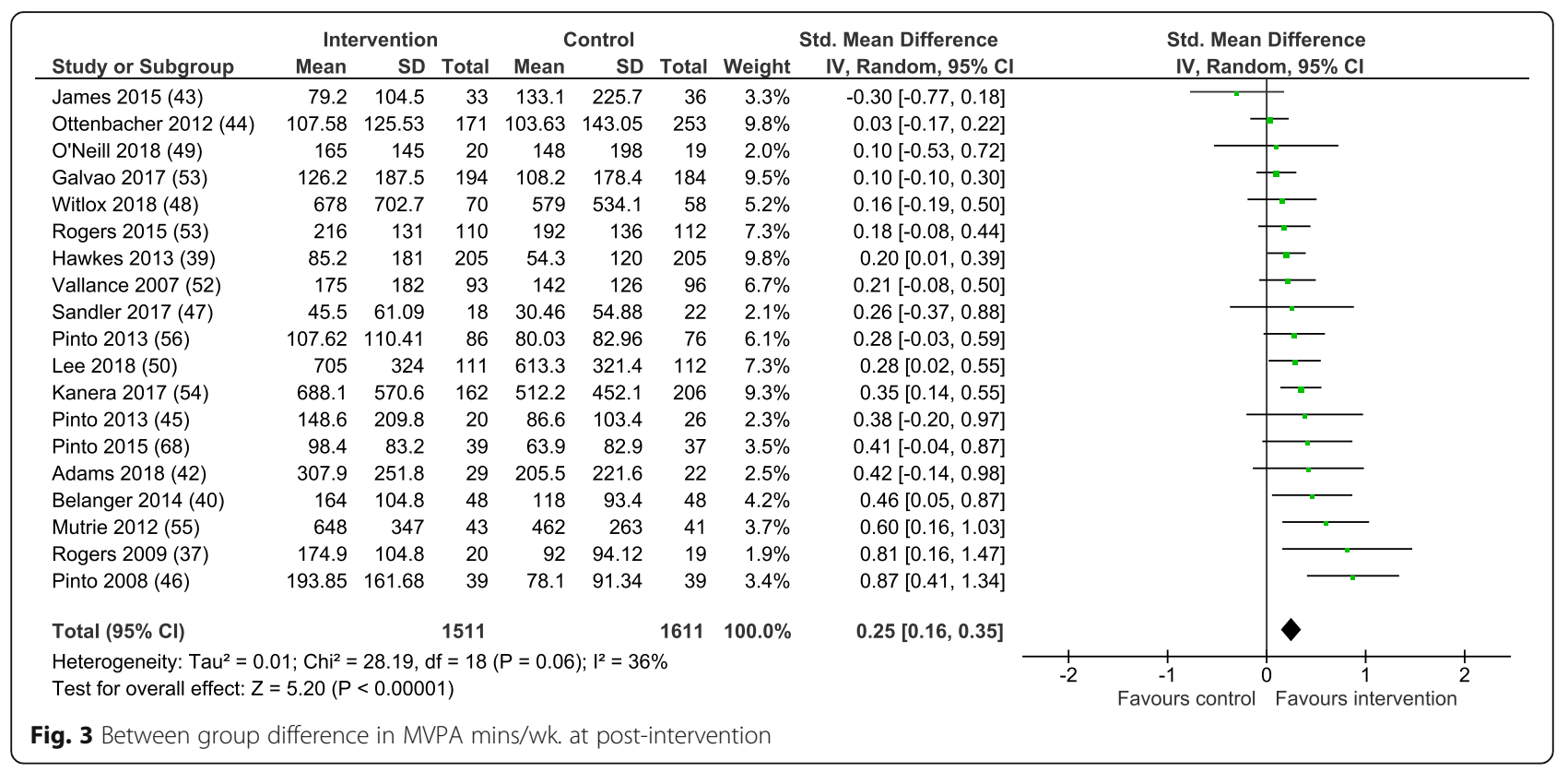

CI $0.15-0.38) \mathrm{I}^{2}=46 \%$, within group pre-post analysis for control groups SMD: 0.23 (95\% CI $0.09-$ 0.38) $\mathrm{I}^{2}=70 \%$, within group pre-post analysis for intervention groups SMD: 0.48 (95\% CI $0.29-0.67) \mathrm{I}^{2}$ $=85 \%$.

\section{BCTs and physical activity outcomes}

Studies included an average of 10.3 BCTs (range 2$20)$, goal setting $(n=25)$, self-monitoring $(n=22)$, instruction on how to perform a behaviour $(n=19)$ and problem solving $(n=18)$ were most frequently reported (see Table 3). There were few notable differences in the BCTs identified within studies when comparing across the classifications 'very promising, 'quite promising' or 'not promising'. The BCTs of 'goal setting', 'problem solving', 'self-monitoring' and 'instruction on how to perform a behaviour' were used often, irrespective of study effectiveness. Additionally the BCTs 'action planning, 'graded tasks' and 'social support (unspecified)' were present in the 'very

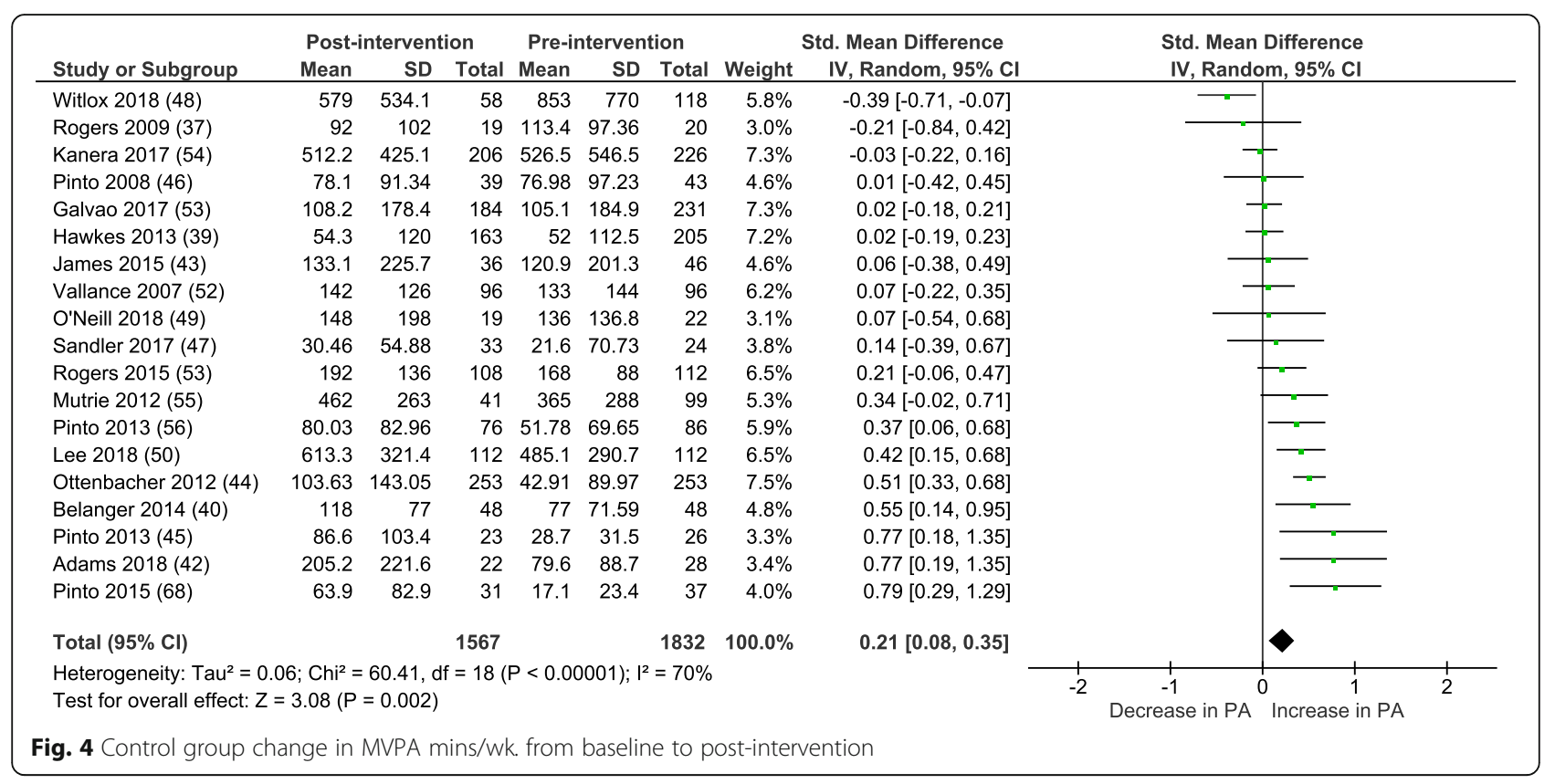




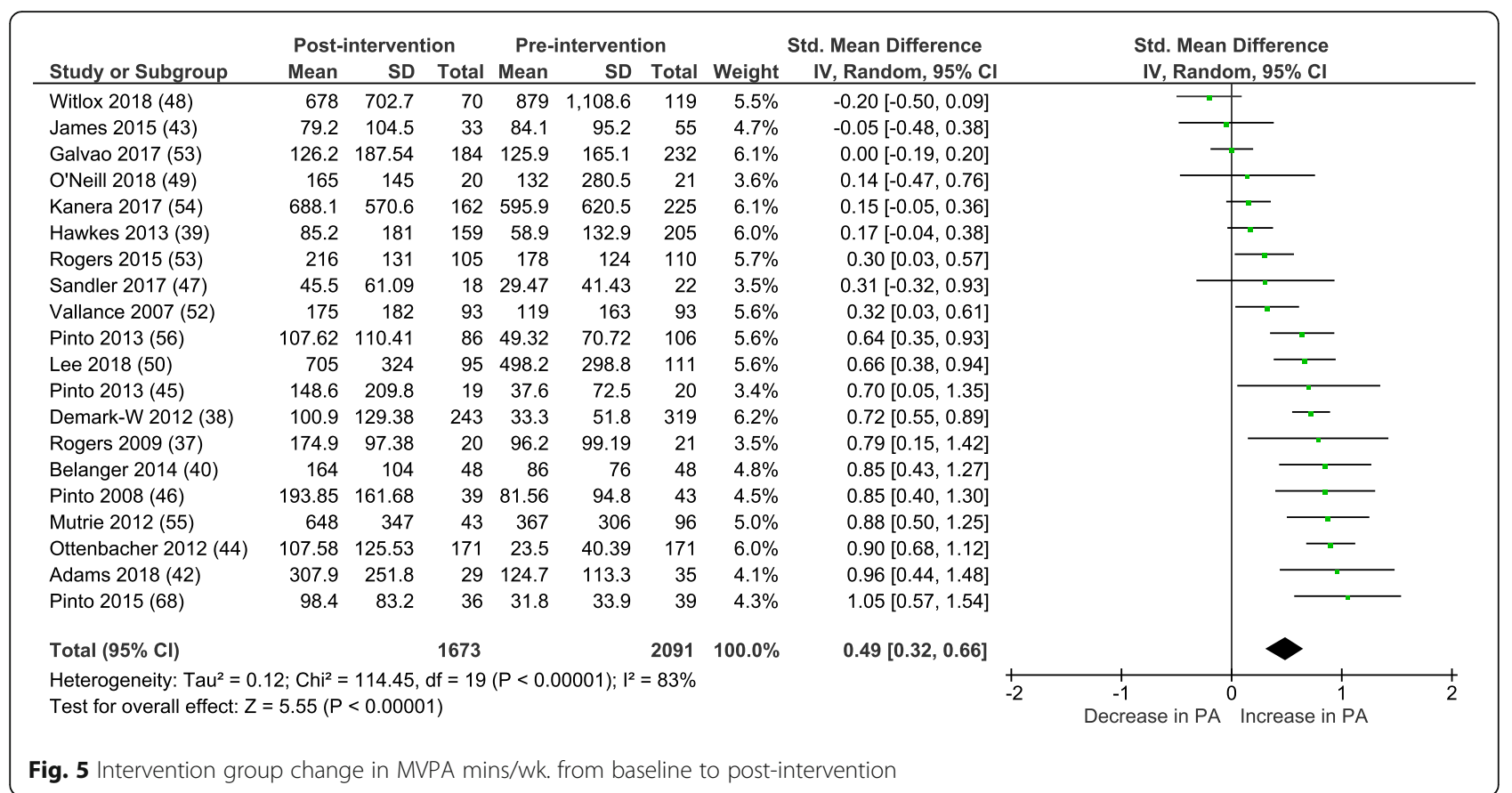

promising' and 'quite promising' studies but most often absent from the 'not promising' studies. When considering the study populations and characteristics as described in Table 1, participants in the studies classified as 'not promising' tended to be older (mean age 63 yrs. versus $57 \mathrm{yrs}$. in the 'very promising' and 'quite promising' studies). Also, supervised physical activity tended not to be provided as part of the intervention in the former studies. There were also fewer contacts with participants during the intervention. Moreover, two of the studies [41, 47] purposefully recruited participants with limitations, pain and fatigue respectively, and two included exclusively prostate cancer patients, many of whom were still undergoing treatment.

When considering physical activity behaviour change in the control groups, four of the eight studies describing significant increases in physical activity from baseline to post intervention follow-up included 'attention control' compared, to only one study that used attention control but did not find any significant change.

\section{Discussion}

This is the first systematic review and meta-analysis to synthesise the evidence from RCTs examining long-term physical activity behaviour change following intervention in cancer populations. With a significant overall SMD (0.25; 95\% CI $=0.16-0.35)$ in MVPA mins/wk. we can conclude interventions achieved a small effect, compared with controls, on long-term physical activity behaviour defined as a minimum 3 month follow-up. Results were similar for studies presenting a minimum 6 month follow-up period. Also unique to this review is the synthesis of evidence of physical activity behaviour change in the control groups with an average increase of approximately 30 mins/wk. MVPA from baseline to post intervention follow-up. This suggests that the standard mean differences between the intervention and control groups may underestimate the impact of the interventions on physical activity behaviour. Indeed, when considering data only from the intervention groups, we saw an average increase of 65.30 (95\% CI = 45.59-85.01) mins/wk. of MVPA at the last follow-up point. Such a change, especially in the intervention groups, may be clinically meaningful. There is evidence that older men who move from being sedentary to engaging in at least light activity have significantly lower risk of all-cause mortality than those who remain sedentary [58]. Furthermore, there is data showing that women with breast cancer who engage in 3-8.9 MET-hrs/wk. of physical activity, had a relative risk of death from breast cancer of 0.50 (95\% CI, 0.31-0.82) compared to 0.80 (CI 0.60-1.06) for those doing less that 3 MET-hours per week, with 3 MET hours equivalent to walking at an average pace of 2 to $2.9 \mathrm{mph}$ for $1 \mathrm{~h}$ [59].

The notion of contamination in control groups in lifestyle interventions is a widely acknowledged limitation of such trials. Steins Bisschop et al. [28] conducted a systematic review of control group design, contamination and drop out in oncology trials. They found that $75 \%$ of studies in their review reported control group contamination. Furthermore, Waters and colleagues [60] found that $28 \%$ of physical 


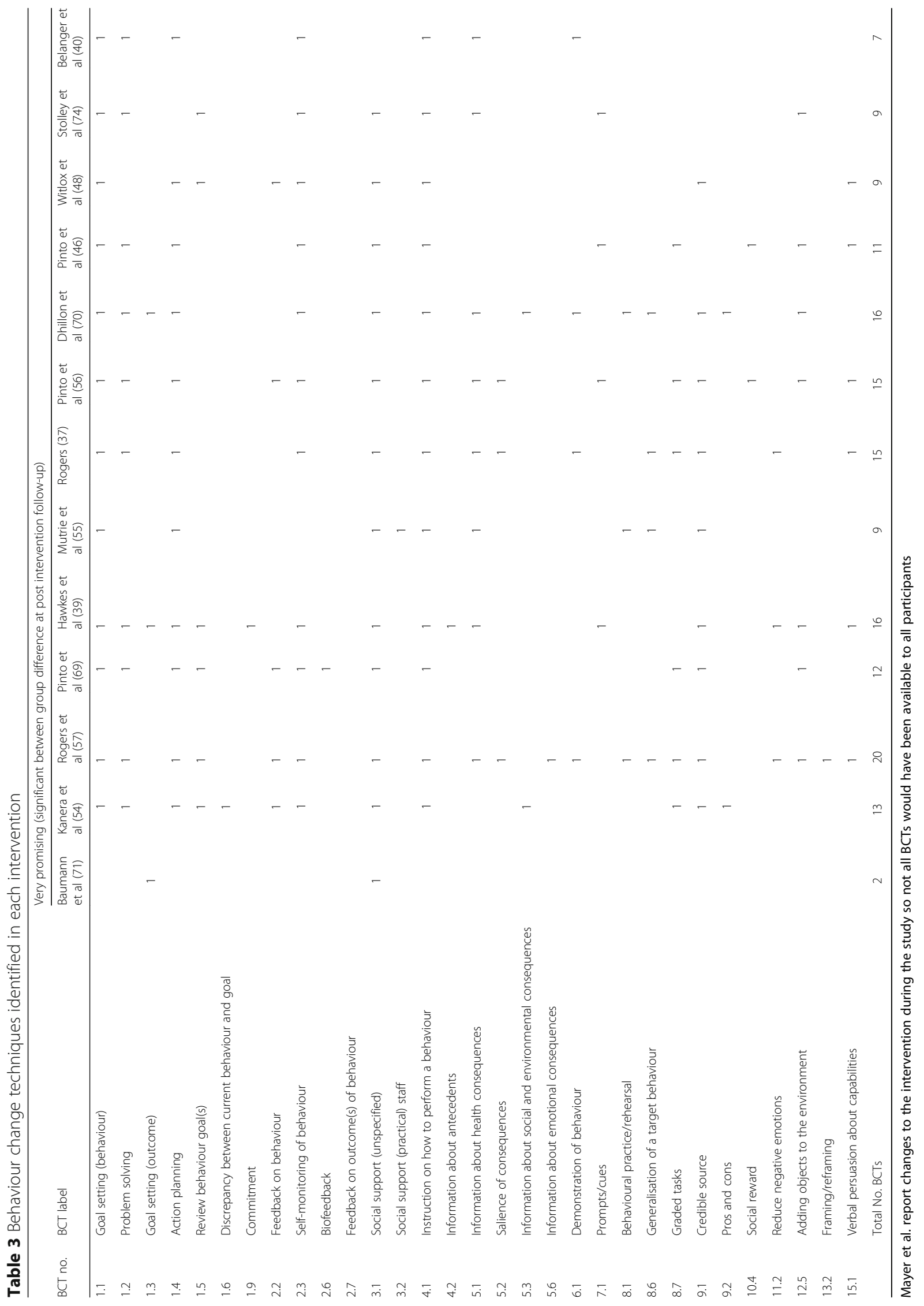




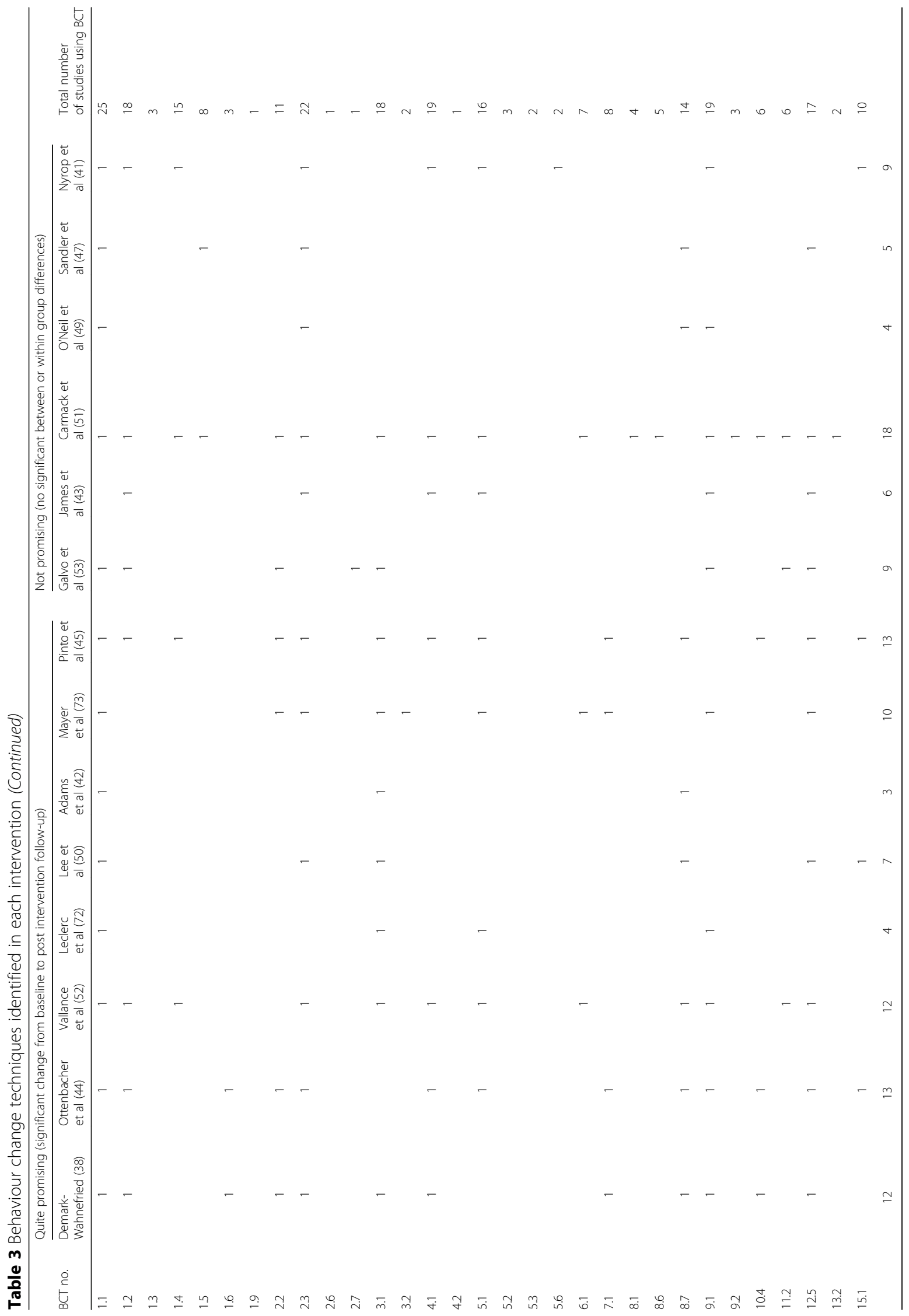


activity interventions reviewed $(n=28$, which are not limited to cancer populations) reported meaningful improvements in physical activity levels of the control groups. However the current review is the first to quantify the degree of this change across cancer trials. Notably, we showed that 7 of the 19 studies (35\%) showed statistically significant improvements in physical activity in control groups at follow-up.

By identifying the BCTs included within the studies we hoped to unpick the key ingredients that may lead to successful, long-term behaviour change in cancer populations. 'Goal setting (behaviour), 'self-monitoring of behaviour,' 'problem solving' and 'instruction on how to perform a behaviour' were frequently reported across studies, irrespective of effectiveness. There were few clear differences in BCTs identified in the 'very promising' and 'quite promising' verses the 'not promising' studies. The exceptions were; 1) 'graded tasks', setting easy tasks and make them increasingly difficult but achievable. 2) 'social support (unspecified)', that is, providing or arranging social support or non-contingent praise or reward for performance of the behaviour; this includes studies that used motivational interviewing techniques. 3) 'action planning', encourage detailed planning of the behaviour e.g. where and when you plan to exercise. A recent review of maintenance of weight loss after cancer report similar findings [61]. Specifically, Hoedjes et al. found that the BCTs of 'goal setting, 'action planning,' 'social support' and 'instruction of how to perform the behaviour' were present in interventions that effectively promoted sustained weight loss. However the authors did not describe the BCTs used in the unsuccessful interventions and therefore no comparisons are made. Similarities can also be seen in a recent review of maintenance of physical activity behaviour change in inactive healthy adults, with a small effect on physical activity behaviour at six months follow-up $(\mathrm{d}=0.21,95 \%$ $\mathrm{CI}=0.12-0.30$ ). Effectiveness was associated with 'action planning,' 'instruction on how to perform the behaviour', 'prompts/cues', 'behaviour practice/rehearsal', 'graded tasks' and 'self-reward' [62].

It appears that inclusion of certain BCTs may increase the likelihood of intervention success, however the striking similarities of BCTs across 'very promising,' 'quite promising' and 'not promising' studies in the current review suggests there are other population or context characteristics that impact on effectiveness.

In general, across all included studies, participants were relatively young, female, well-educated and predominantly white. Recruitment rates were variable but typically low, suggesting an amotivated population and/or strict inclusion/exclusion criteria. It appears therefore that current interventions reflect and reinforce structural societal inequalities. Participants in the studies that did not produce any notable change in behaviour tended to be older, two comprised exclusively prostate cancer populations and two included those with pain and clinically significant fatigue. As such, it may be that a stepped care approach to behaviour change is needed. This finding is supported by Morey and colleagues [63] who performed group trajectory analysis for patients participating in the RENEW study, a distanced-based multimodal lifestyle interventions in older long-term cancer survivors. They found that patients who remained inactive throughout the study had low levels of physical function at baseline which continued to decline over time. This is in contrast to those who achieved marked improvement in physical activity throughout the 12-month intervention and 12-month follow-up periods who reported considerably higher levels of physical function on study entry. By providing stratified support, offering more intensive interventions and one-to-one support to those who need it most, a more representative population of cancer patients may be encouraged to engage in positive behaviour change which will be more likely to affect change. Such an approach is supported by a recent Individual Patient Data meta-analysis examining the moderator effect of baseline values on the exercise outcomes of fatigue, aerobic fitness, muscle strength, quality of life and physical function [64]. Moreover, Buffart and colleagues found that for patients who had completed cancer treatment, those with worst baseline QoL, fatigue and physical function experienced the largest improvements following exercise intervention, suggesting the greatest impact of interventions may be seen by targeting those most in need.

Evidence presented here suggests that motivated, well-educated, younger and white patients may achieve a clinically important increase in their long-term physical activity behaviour with a relatively low-intensity intervention. This is supported by the observation of increases in physical activity in the control groups who were typically provided with brief written information. Nonetheless, inclusion of a supervised component and frequent contact with participants may further increase intervention effectiveness. This is corroborated by the finding that four of the eight studies that found significant improvements in physical activity within the control group's used a contact control study design, so frequent contact may have prompted participants to become active. Similar conclusions were drawn in a recent review by Bluethmann et al. [18]. In their synthesis of 14 RCTs aimed at increasing physical activity in breast cancer survivors they explored the effect of intervention intensity (i.e. number of intervention sessions) on 
behaviour change. They found that higher intensity interventions tended to produce larger effects, but some of the largest effects came from interventions they had categorised as 'medium' intensity, including home-based programmes with telephone support. This is supported by an earlier review of broad reach interventions, concluding telephone, print and web-based interventions were effective in initiating behaviour change [31]. However, both reviews found limited evidence for maintenance of change. Finally, in a review of maintenance of behaviour change, although not limited to cancer populations, Fjeldsoe et al. reported that physical activity and dietary interventions with more intervention contacts were more likely to achieve maintenance of behaviour change.

Another important consideration is that participants choosing to enrol in the physical activity interventions tended to already be engaging in some physical activity. Of the 19 studies included in the between groups meta-analysis, 12 excluded participants who were already meeting physically active guidelines; however the median baseline levels of physical activity in the intervention groups was 86 mins/wk. of MVPA. It is likely that mechanisms of behaviour change are different for those who are engaging in no physical activity at all versus those who are somewhat active. As such alternative intervention methods may be required if targeting the more inactive and sedentary populations. This is of utmost importance given that population health benefits may be achieved by supporting those who are sedentary to becoming moderately active [65]. Also, the dose response relationship between physical activity and health benefits, particularly in cardiovascular disease, supports the message that 'some physical activity is better than none' [66] and older adults who participate in any amount of physical activity will see some health benefits [67].

It is important that the results of this paper are interpreted with some caution. The meta-analysis relied almost exclusively on data collected from self-reported physical activity measures, which are known to have poor correlation with objective measures of physical activity. There was also considerable variation in outcome measures used across studies. This may explain in part the wide range in baseline levels of physical activity and high heterogeneity reported in the within-group meta-analysis. When considering conclusions regarding $\mathrm{BCTs}$ associated with effectiveness, these findings are limited by the incomplete reporting of interventions and their components. Furthermore, the guidelines for coding of BCTs are very stringent so it is possible that BCTs embedded in interventions are not always captured by the coding if descriptions are not sufficiently precise. When describing interventions in future studies, we suggest authors refer to Michie et al's [22] coding scheme to ensure BCTs are appropriately documented and thus accurately coded in future efforts of data synthesis. Finally, it is possible that our method of trial classification as 'very promising,' 'quite promising' or 'not promising' may result in false negatives if studies are not powered to detect statistical differences in physical activity.

\section{Conclusions}

This is the first systematic review and meta-analysis of physical activity maintenance across cancer types. When considering differences between intervention and comparison groups, small differences are evident in favour of intervention groups. Improvements in physical activity behaviour in control groups suggest these between group analysis may underestimate intervention effect. Meta-analysis of change in activity levels in intervention groups indicates a clinically significant mean increase of over $1 \mathrm{~h}$ per week at post intervention follow-up. Analysis of intervention components and context suggests reasonably low-intensity interventions may be sufficient in prompting lasting behaviour change in motivated, young, well educated and white populations but that more intensive support is likely to be required for other populations, especially for older people and those with physical limitations. Future interventions should seek to encourage engagement from more representative samples including older adults, those from ethnic minorities and less educated backgrounds. A stepped care approach to intervention design and delivery may enable effective use of limited resources with additional support provided to those most in need.

\section{Additional file}

Additional file 1: Medline search strategy. (DOCX 18 kb)

\section{Acknowledgements}

We would like to thank Dr. Sally Wheelwright for her advice regarding methods, data acquisition and analysis, and Karen Welch for supporting the development of search terms and executing the searches.

\section{Funding}

Chloe Grimmett is funded by a National Institute for Health Research (NIHR) Post-Doctoral Fellowship for this research project. This publication presents the independent research funded by the NIHR. The views expressed are those of the authors and not necessarily those of the NHS, the NIHR or the Department of Health and Social Care. No funding bodies had any role in the design of the study, collection, analysis, and interpretation of data or in the writing of the manuscript.

Availability of data and materials

All data generated or analysed during this study are included in this published article [and its supplementary information files].

Authors' contributions

CG made substantial contributions to the conception, design, acquisition of data, analysis, interpretation and drafted the manuscript. TC made substantial 
contributions to the acquisition of data, interpretation and contributed critical revisions to the manuscript. JB made substantial contributions to the conception and design of the review and contributed critical revisions to the manuscript. JS made substantial contributions to data analysis and interpretation and contributed critical revisions to the manuscript. BP made substantial contributions to the conception and design of the review and contributed critical revisions to the manuscript. CM made substantial contributions to the conception and design of the review and contributed critical revisions to the manuscript. CF made substantial contributions to the conception and design of the review and contributed critical revisions to the manuscript. All authors read and approved the final manuscript.

\section{Ethics approval and consent to participate}

Not applicable.

\section{Consent for publication}

Not applicable.

\section{Competing interests}

The authors declare that they have no competing interests.

\section{Publisher's Note}

Springer Nature remains neutral with regard to jurisdictional claims in published maps and institutional affiliations.

\section{Author details}

${ }^{1}$ School of Health Sciences, University of Southampton, Southampton, UK. ${ }^{2}$ Southampton Health Technology Assessments Centre, University of Southampton, Southampton, UK. ${ }^{3}$ Faculty of Health Sciences, University of Ottawa, Ottawa, ON, Canada. ${ }^{4}$ College of Nursing, University of South Carolina, Columbia, USA. ${ }^{5}$ Faculty of Public Health and Policy, London School of Hygiene and Tropical Medicine, London, UK.

\section{Received: 26 November 2018 Accepted: 4 March 2019} Published online: 27 April 2019

\section{References}

1. UK CR. World Wide Cancer Incidence Statistics 2014 [Available from: https:// www.cancerresearchuk.org/health-professional/cancer-statistics/worldwidecancer/incidence\#heading-Two.

2. UK CR. World Cancer Factsheet 2014 [Available from: https://www. cancerresearchuk.org/sites/default/files/cs_report_world.pdf.

3. Stein KD, Syrjala KL, Andrykowski MA. Physical and psychological long-term and late effects of cancer. Cancer. 2008;112(11 Suppl):2577-92.

4. Fong DYT, Ho JWC, Hui BPH, Lee AM, Macfarlane DJ, Leung SSK, et al. Physical activity for cancer survivors: meta-analysis of randomised controlled trials. BMJ. 2012;344:e70.

5. Meneses-Echávez JF, González-Jiménez E, Ramírez-Vélez R. Effects of supervised exercise on cancer-related fatigue in breast cancer survivors: a systematic review and meta-analysis. BMC Cancer. 2015;15(1):77.

6. Blair SN, Morris JN. Healthy hearts-and the universal benefits of being physically active: physical activity and health. Ann Epidemiol. 2009;19(4):253-6.

7. Lahart IM, Metsios GS, Nevill AM, Carmichael AR. Physical activity, risk of death and recurrence in breast cancer survivors: a systematic review and meta-analysis of epidemiological studies. Acta Oncol. 2015;54(5):635-54.

8. Schmid D, Leitzmann MF. Association between physical activity and mortality among breast cancer and colorectal cancer survivors: a systematic review and meta-analysis. Ann Oncol. 2014;25(7):1293-311.

9. Van Blarigan EL, Meyerhardt JA. Role of Physical Activity and Diet After Colorectal Cancer Diagnosis. J Clin Oncol. 2015;33(16):1825.

10. Wiseman M. The second World Cancer Research Fund/American Institute for Cancer Research expert report. Food, nutrition, physical activity, and the prevention of cancer: a global perspective. Proc Nutr Soc. 2008;67(3):253-6.

11. Rock CL, Doyle C, Demark-Wahnefried W, Meyerhardt J, Courneya KS, Schwartz AL, et al. Nutrition and physical activity guidelines for Cancer survivors. Ca-a Cancer Journal for Clinicians. 2012:62(4):243-74.

12. Schmitz KH, Courneya KS, Matthews C, Demark-Wahnefried W, Galvao DA, Pinto BM, et al. American College of Sports Medicine roundtable on exercise guidelines for Cancer survivors. Med Sci Sports Exerc. 2010;42(7):1409-26.
13. Irwin $M L$, McTiernan A, Bernstein L, Gilliland FD, Baumgartner $R$, Baumgartner $\mathrm{K}$, et al. Physical activity levels among breast cancer survivors. Med Sci Sports Exerc. 2004;36(9):1484-91.

14. Williams K, Steptoe A, Wardle J. Is a cancer diagnosis a trigger for health behaviour change? Findings from a prospective, population-based study. $\mathrm{Br}$ J Cancer. 2013;108(11):2407-12

15. Grimmett C, Wardle J, Steptoe A. Health behaviours in older cancer survivors in the English longitudinal study of ageing. Eur J Cancer. 2009; 45(12):2180-6.

16. Wang Z, McLoone P, Morrison DS. Diet, exercise, obesity, smoking and alcohol consumption in cancer survivors and the general population: a comparative study of 16282 individuals. Br J Cancer. 2015;112(3):572-5.

17. Stacey FG, James EL, Chapman K, Courneya KS, Lubans DR. A systematic review and meta-analysis of social cognitive theroy-based physical activity and/or nutrition behavior change interventions for cancer survivors. J Cancer Surviv. 2015;9(2):305-38.

18. Bluethmann SM, Vernon SW, Gabriel KP, Murphy CC, Bartholomew LK. Taking the next step: a systematic review and meta-analysis of physical activity and behavior change interventions in recent post-treatment breast cancer survivors. Breast Cancer Res Treat. 2015;149(2):331-42.

19. Fjeldsoe B, Neuhaus M, Winkler E, Eakin E. Systematic review of maintenance of behavior change following physical activity and dietary interventions. Health Psychol. 2011;30(1):99-109.

20. Jankowski CM, Ory MG, Friedman DB, Dwyer A, Birken SA, Risendal B. Searching for maintenance in exercise interventions for cancer survivors. J Cancer Surviv. 2014;8(4):697-706.

21. Davidson KW, Goldstein M, Kaplan RM, Kaufmann PG, Knatterud GL, Orleans $C T$, et al. Evidence-based behavioral medicine: what is it and how do we achieve it? Ann Behav Med. 2003;26(3):161-71.

22. Michie S, Richardson M, Johnston M, Abraham C, Francis J, Hardeman W, et al. The behavior change technique taxonomy (v1) of 93 hierarchically clustered techniques: building an international consensus for the reporting of behavior change interventions. Ann Behav Med. 2013;46(1):81-95.

23. Bishop FL, Fenge-Davies AL, Kirby S, Geraghty AW. Context effects and behaviour change techniques in randomised trials: a systematic review using the example of trials to increase adherence to physical activity in musculoskeletal pain. Psychol Health. 2015;30(1):104-21.

24. Cradock KA, ÓLaighin G, Finucane FM, Gainforth HL, Quinlan LR, Ginis KAM. Behaviour change techniques targeting both diet and physical activity in type 2 diabetes: a systematic review and meta-analysis. Int J Behav Nutr Phys Act. 2017;14(1):18.

25. Nyman SR, Adamczewska N, Howlett N. Systematic review of behaviour change techniques to promote participation in physical activity among people with dementia. Br J Health Psychol. 2018;23(1):148-70.

26. Michie S, Abraham C, Whittington C, McAteer J, Gupta S. Effective techniques in healthy eating and physical activity interventions: a metaregression. Health Psychol. 2009;28(6):690-701.

27. Hoffmann TC, Glasziou PP, Boutron I, Milne R, Perera R, Moher D, et al. Better reporting of interventions: template for intervention description and replication (TIDieR) checklist and guide. BMJ. 2014;348:g1687.

28. Steins Bisschop CN, Courneya KS, Velthuis MJ, Monninkhof EM, Jones LW, Friedenreich $C$, et al. Control group design, contamination and drop-out in exercise oncology trials: a systematic review. PLoS One. 2015;10(3):e0120996.

29. Centre for Reviews and Dissemination. Systematic reviews: CRD's guidance for undertaking reviews in health care. Third ed. University of York: York Publishing Services Ltd: 2009.

30. Moher D, Liberati A, Tetzlaff J, Altman DG. Preferred reporting items for systematic reviews and meta-analyses: the PRISMA statement. BMJ. 2009; 339:b2535.

31. Goode AD, Lawler SP, Brakenridge CL, Reeves MM, Eakin EG. Telephone, print, and web-based interventions for physical activity, diet, and weight control among cancer survivors: a systematic review. J Cancer Surviv. 2015;9(4):660-82.

32. Spark LC, Reeves MM, Fjeldsoe BS, Eakin EG. Physical activity and/or dietary interventions in breast cancer survivors: a systematic review of the maintenance of outcomes. J Cancer Surviv. 2013;7(1):74-82

33. Higgins JPT, Altman DG, Gøtzsche PC, Jüni $P$, Moher D, Oxman AD, et al. The Cochrane Collaboration's tool for assessing risk of bias in randomised trials. BMJ. 2011;343:d5928.

34. Review Manager (RevMan) [computer program]. Version 5.3 Copenhagen: The Nordic Cochrane Centre, The Cochrane Collaboration, 2014. 
35. Roberts AL, Fisher A, Smith L, Heinrich M, Potts HWW. Digital health behaviour change interventions targeting physical activity and diet in cancer survivors: a systematic review and meta-analysis. J Cancer Surviv. 2017;11(6):704-19.

36. Gardner B, Smith L, Lorencatto F, Hamer M, Biddle SJ. How to reduce sitting time? A review of behaviour change strategies used in sedentary behaviour reduction interventions among adults. Health Psychol Rev. 2016;10(1):89-112

37. Rogers LQ, Hopkins-Price P, Vicari S, Markwell S, Pamenter R, Courneya KS, et al. Physical activity and health outcomes three months after completing a physical activity behavior change intervention: persistent and delayed effects. Cancer Epidemiol Biomark Prev. 2009;18(5):1410-8.

38. Demark-Wahnefried W, Morey MC, Sloane R, Snyder DC, Miller PE, Hartman TJ, et al. Reach out to enhance wellness home-based diet-exercise intervention promotes reproducible and sustainable long-term improvements in health behaviors, body weight, and physical functioning in older, overweight/obese cancer survivors. J Clin Oncol. 2012;30(19):2354-61.

39. Hawkes AL, Chambers SK, Pakenham KI, Patrao TA, Baade PD, Lynch BM, et al. Effects of a telephone-delivered multiple health behavior change intervention (CanChange) on health and behavioral outcomes in survivors of colorectal cancer: a randomized controlled trial. J Clin Oncol. 2013;31(18):2313-21.

40. Belanger L, Mummery WK, Clark AM, Courneya KS. Effects of targeted print materials on physical activity and quality of life in young adult cancer survivors during and after treatment: an exploratory randomized controlled trial. J Adolescent Young Adult Oncol. 2014;3(2):83-91.

41. Nyrop KA, Callahan LF, Cleveland RJ, Arbeeva LL, Hackney BS, Muss HB. Randomized controlled trial of a home-based walking program to reduce moderate to severe aromatase inhibitor-associated arthralgia in breast Cancer survivors. Oncologist. 2017;11:11

42. Adams SC, Delorey DS, Davenport MH, Fairey AS, North S, Courneya KS. Effects of high-intensity interval training on fatigue and quality of life in testicular cancer survivors. Br J Cancer. 2018;118(10):1313-21.

43. James EL, Stacey FG, Chapman K, Boyes AW, Burrows T, Girgis A, et al. Impact of a nutrition and physical activity intervention (ENRICH: exercise and nutrition routine improving Cancer health) on health behaviors of cancer survivors and carers: a pragmatic randomized controlled trial. BMC Cancer. 2015;15:710.

44. Ottenbacher AJ, Day RS, Taylor WC, Sharma SV, Sloane R, Snyder DC, et al. Long-term physical activity outcomes of home-based lifestyle interventions among breast and prostate cancer survivors. Support Care Cancer. 2012; 20(10):2483-9.

45. Pinto BM, Papandonatos GD, Goldstein MG, Marcus BH, Farrell N. Homebased physical activity intervention for colorectal cancer survivors. Psychooncology. 2013;22(1):54-64.

46. Pinto BM, Rabin C, Papandonatos GD, Frierson GM, Trunzo JJ, Marcus BH. Maintenance of effects of a home-based physical activity program among breast cancer survivors. Support Care Cancer. 2008;16(11):1279-89.

47. Sandler CX, Goldstein D, Horsfield S, Bennett BK, Friedlander M, Bastick PA, et al. Randomized evaluation of cognitive-behavioral therapy and graded exercise therapy for post-cancer fatigue. J Pain Symptom Manag. 2017;54(1):74-84.

48. Witlox L, Hiensch AE, Velthuis MJ, Bisschop CNS, Los M, Erdkamp FLG, et al. Four-year effects of exercise on fatigue and physical activity in patients with cancer. BMC Med. 2018;16:9.

49. O'Neill LM, Guinan E, Doyle SL, Bennett AE, Murphy C, Elliott JA, et al. The RESTORE randomized controlled trial: impact of a multidisciplinary rehabilitative program on cardiorespiratory fitness in Esophagogastric cancer survivorship. Ann Surg. 2018;12:12.

50. Lee CF, Ho JWC, Fong DYT, Macfarlane DJ, Cerin E, Lee AM, et al. Dietary and physical activity interventions for colorectal Cancer survivors: a randomized controlled trial. Sci Rep. 2018;8(1):5731.

51. Carmack Taylor CL, Demoor C, Smith MA, Dunn AL, Basen-Engquist K, Nielsen I, et al. Active for life after Cancer: a randomized trial examining a lifestyle physical activity program for prostate cancer patients. Psychooncology. 2006;15(10):847-62.

52. Vallance JK, Courneya KS, Plotnikoff RC, Dinu I, Mackey JR. Maintenance of physical activity in breast cancer survivors after a randomized trial. Med Sci Sports Exerc. 2008;40(1):173-80.

53. Galvao DA, Newton RU, Girgis A, Lepore SJ, Stiller A, Mihalopoulos C, et al. Randomized controlled trial of a peer led multimodal intervention for men with prostate cancer to increase exercise participation. Psychooncology. 2018;27(1):199-207.
54. Kanera IM, Willems RA, Bolman CA, Mesters I, Verboon P, Lechner L. Longterm effects of a web-based cancer aftercare intervention on moderate physical activity and vegetable consumption among early cancer survivors: a randomized controlled trial. Int J Behav Nutr Phys Act. 2017;14(1):19.

55. Mutrie N, Campbell A, Barry S, Hefferon K, McConnachie A, Ritchie D, et al. Five-year follow-up of participants in a randomised controlled trial showing benefits from exercise for breast cancer survivors during adjuvant treatment. Are there lasting effects? J Cancer Surviv. 2012;6(4):420-30.

56. Pinto BM, Papandonatos GD, Goldstein MG. A randomized trial to promote physical activity among breast cancer patients. Health Psychol. 2013;32(6):616-26.

57. Rogers LQ, Courneya KS, Anton PM, Hopkins-Price P, Verhulst S, Vicari SK, et al. Effects of the BEAT Cancer physical activity behavior change intervention on physical activity, aerobic fitness, and quality of life in breast cancer survivors: a multicenter randomized controlled trial. Breast Cancer Res Treat. 2015:149(1):109-19.

58. Wannamethee SG, Shaper AG, Walker M. Changes in physical activity, mortality, and incidence of coronary heart disease in older men. Lancet. 1998;351(9116):1603-8.

59. Holmes MD, Chen WY, Feskanich D, Kroenke CH, Colditz GA. Physical activity and survival after breast cancer diagnosis. Jama. 2005;293(20): 2479-86.

60. Waters L, Reeves M, Fjeldsoe B, Eakin E. Control group improvements in physical activity intervention trials and possible explanatory factors: a systematic review. J Phys Act Health. 2012;9(6):884-95.

61. Hoedjes M, van Stralen MM, Joe STA, Rookus M, van Leeuwen F, Michie S, et al. Toward the optimal strategy for sustained weight loss in overweight cancer survivors: a systematic review of the literature. J Cancer Surviv. 2017; 11(3):360-85.

62. Howlett N, Trivedi D, Troop NA, Chater AM. Are physical activity interventions for healthy inactive adults effective in promoting behavior change and maintenance, and which behavior change techniques are effective? A systematic review and meta-analysis. Transl Behav Med. 2018.

63. Morey MC, Blair CK, Sloane R, Cohen HJ, Snyder DC, Demark-Wahnefried W. Group trajectory analysis helps to identify older cancer survivors who benefit from distance-based lifestyle interventions. Cancer. 2015;121(24): 4433-40.

64. Buffart LM, Sweegers MG, May AM, Chinapaw MJ, van Vulpen JK, Newton $\mathrm{RU}$, et al. Targeting exercise interventions to patients with Cancer in need: an individual patient data meta-analysis. J Natl Cancer Inst. 2018;110(11): 1190-200.

65. Blair SN, Connelly JC. How much physical activity should we do? The case for moderate amounts and intensities of physical activity. Res Q Exerc Sport. 1996;67(2):193-205.

66. Sattelmair J, Pertman J, Ding EL, Kohl HW 3rd, Haskell W, Lee IM. Dose response between physical activity and risk of coronary heart disease: a meta-analysis. Circulation. 2011;124(7):789-95.

67. Chodzko-Zajko WJ, Proctor DN, Fiatarone Singh MA, Minson CT, Nigg CR, Salem GJ, et al. American College of Sports Medicine position stand. Exercise and physical activity for older adults. Med Sci Sports Exerc. 2009; 41(7):1510-30.

68. Pinto BM, Stein K, Dunsiger S. Peers promoting physical activity among breast cancer survivors: a randomized controlled trial. Health Psychol. 2015; 34(5):463-72.

69. Dhillon HM, Bell ML, van der Ploeg HP, Turner JD, Kabourakis M, Spencer L, et al. Impact of physical activity on fatigue and quality of life in people with advanced lung Cancer: a randomised controlled trial. Ann Oncol. 2017;28:28.

70. Baumann FT, Bieck O, Oberste M, Kuhn R, Schmitt J, Wentrock S, et al. Sustainable impact of an individualized exercise program on physical activity level and fatigue syndrome on breast cancer patients in two German rehabilitation centers. Support Care Cancer. 2017;25(4):1047-54.

71. Leclerc AF, Slomian J, Jerusalem G, Coucke P, Bury T, Deflandre D, et al. Exercise and education program after breast Cancer: benefits on quality of life and symptoms at 3, 6, 12, and 24 Months' follow-up. Clin Breast Cancer. 2018; 5(5):e1189-204.

72. Mayer DK, Landucci G, Awoyinka L, Atwood AK, Carmack CL, DemarkWahnefried W, et al. SurvivorCHESS to increase physical activity in colon cancer survivors: can we get them moving? J Cancer Surviv. 2018;12(1):82-94.

73. Stolley M, Sheean P, Gerber B, Arroyo C, Schiffer L, Banerjee A, et al. Efficacy of a Weight Loss Intervention for African American Breast Cancer Survivors. J Clin Oncol. 2017:35(24):2820. 